\title{
Storage and processing working memory functions in Alzheimer-type dementia
}

\author{
T. Vecchi ${ }^{\mathrm{a}, \mathrm{b} *}$, V. Saveriano ${ }^{\mathrm{b}, \mathrm{c}}$ and L. Paciaroni ${ }^{\mathrm{c}}$ \\ ${ }^{a}$ Istituto di Psicologia, Universita di Pavia, Italy \\ ${ }^{\mathrm{b}}$ Dipartimento di Psicologia Generale, Universita \\ di Padova, Italy \\ ${ }^{\mathrm{c}}$ Unita di Neurologia, Ospedale Geriatrico, INRCA, \\ Ancona, Italy
}

\begin{abstract}
A selective deterioration of working memory functions has been suggested as an explanation of the cognitive decay occurring in normal ageing as well as in Alzheimer-type dementia. Recent studies have highlighted that elderly people's limitations in working memory functions may be better interpreted when analysing the specific characteristics of the cognitive process (i.e., passive storage or active manipulation of information). In the present study, we have adapted a procedure used to investigate age-related memory modifications, involving both verbal and visuo-spatial material in tasks tapping passive and active processes, to investigate the deterioration associated with Alzheimer's disease. A group of Alzheimer patients in the early stages of the disease were matched to a control group of healthy elderly. Results show that Alzheimer patients performed less accurately than the control group in all tasks. However, the deficit was maximised in the case of active processes, regardless of the type of material used (verbal or visuo-spatial). These data highlight the importance of considering the amount of active processing as the key variable when interpreting the decay in cognitive functions in the early stages of Alzheimer's disease.
\end{abstract}

Keywords: Working memory, Alzheimer-type dementia

\section{Introduction}

The working memory system [3] is characterised by the integration of both storage and processing components in a unique cognitive structure. However, empirical studies devoted to investigating the architecture of this system have been mainly directed at distinguishing between components of the system associated with the specific type of material that could be processed. The functional dissociation between verbal and visuo-spatial components (e.g., $[11,23])$ or between visual and spatial components (e.g., $[8,13])$ has been repeatedly confirmed. However, little attention

${ }^{*}$ Corresponding author: Tomaso Vecchi, Istituto di Psicologia, Università di Pavia, P.za Botta 6, 27100 Pavia, Italy. Fax: +39 0382506272; E-mail: tomaso.vecchi@unimi.it. has been paid to analyse working memory processes in terms of the specific amount of storage or processing requirements and, consequently, to propose a distinction between passive storage and active processing components within the working memory system. Within such a framework, each task could be identified by a proportion of storage and processing functions. Storage refers to the maintenance of information in the same format as it was previously encoded, and processing refers to the modification, integration, or transformation which may be required by the task (see [24]).

The deterioration of working memory in Alzheimertype dementia (DAT) has been observed repeatedly (e.g., [21]) and studies carried out using a dual task paradigm have shown a selective deterioration of the Alzheimer group under these conditions [4]. This has led to a deficit in the central executive component of the working memory system being proposed. The effect of dual task procedures has been confirmed by the same authors in a successive study [2], also allowing them to exclude possible artefacts due to task complexity.

Several empirical investigations have addressed the possibility that central executive processes can be characterised in terms of shifting of attention or, more in general, in terms of an amalgam of control mechanisms underlying WM functions (e.g., [15]). These processes are implicated in the concurrent execution of more than one task at the same time and DAT patients are clearly impaired in dual-task procedures (e.g., $[10,12,14,16])$. Within this theoretical framework, the WM central executive component can be considered as an external attentional mechanisms (see [19]) that does not play a direct role in the actual execution of the task. Patients' impairments is then associated to a dysfunction in these control structures.

Another way of characterising central executive functions is to refer to the specific nature of each single task, in terms of amount of integration/transformation of information that is required. In particular, the passive-active distinction could be important in interpreting WM functions and in assessing DAT patients. This distinction has demonstrated to be relevant in ex- 
plaining specific pattern of WM functions in a number of individual differences studies. However, the relationship between passive and active processes cannot be easily interpreted within a traditional working memory framework. In fact, both passive and active processes are involved, to different extents, in the execution of the various tasks. Recent neuroimaging findings have clearly demonstrated the existence of separate neural mechanisms for storing or processing information (see [20]). However, it has also been suggested that, from a theoretical point of view, passive and active processing structures could be interpreted along a continuum reflecting the extent to which active processes are required by each task. This theoretical interpretation seems to better represent the functioning of the WM system by clarifying the continuum dimension between modality specific and process-specific structures (see [7]). The need for a theoretical framework that specify the relationship between passive and active functions does not exclude that, in principle, the two opposite pole of the continuum could be associated with the existence of pure passive or active tasks. In particular, there has been recently a strong effort to develop specific tools to measure active processing while minimising passive storage [26], and empirical results do support the independence of active manipulation processes. In other words, active processes are not necessarily associated with passive storage, but could be based on perceptual stimuli only, thus minimally depending on short-term maintenance of information.

In the present study, we adapted the procedure used by [24] to investigate age differences in working memory by comparing a group of DAT patients in an early stage of the disease with a control group of healthy elderly. Four tasks were used: Two passive tasks (one verbal and one visuo-spatial) and two active tasks (one verbal and one visuo-spatial).

\section{Methods}

\subsection{Participants}

Sixteeen AD patients, aged between 63 and 74 years (mean age $=70.1$, sd $=3.4$ ), 8 males and 8 females, participated in this study. They were recruited through the Alzheimer Center of the Geriatric Hospital of Ancona, Italy. All have been diagnosed as affected by Alzheimer-type dementia on the basis of clinical history, neurological examination and neuropsychological assessment. AD patients met the criteria for di- agnosis of probable Alzheimer-type dementia (DSM [1]). Moreover, criterion for inclusion in the experimental group was a score between 21 and 24 for the Mini Mental State Examination (MMSE [9]), thus indicating a mild cognitive impairment. Sixteen elderly subjects were selected as control group, matched for age (aged between 63 and 75 years, mean age $=69.3$, $\mathrm{sd}=3.8$ ), gender ( 8 males and 8 females), and years of education and type of school. We also excluded from our samples subjects whose working activities could have led to a specific advantage for the execution of the experimental tasks, such as architects or graphic designers (see [18]). None of the subjects had either a record of neurological disease or presented pathologies of the central nervous system, other than the Alzheimer's disease in the experimental group.

\subsection{Materials and procedure}

Four tasks were used: a verbal passive task, a verbal active task, a visuo-spatial passive task and a visuospatial active task. All tasks were presented in a span version requiring subjects to solve at least two items at each level of complexity in order to proceed to the following level. Three span sequences were designed for each level of complexity. The order of presentation of the different material (verbal or visuo-spatial), for the different types of task (passive or active), were counterbalanced across subjects thus obtaining 4 different orders.

Verbal passive task: Verbal span task. Participants had to remember lists of bisyllabic words of increasing length. Words were read by the experimenter at a 2 -sec. rate. The Italian version of this test has been standardized by [22].

Verbal active task: Nonword generation task [25]. Sequences of syllables of increasing length were read by the experimenter at a 1 -sec. rate. For each sequence, participants had to generate the nonword resulting by stringing together the first letter of each syllable. For example a sequence at level 4 could consist of 'pu, ite, fu, oto' and the subject had to generate the nonword 'pifo'.

Visuo-spatial passive task: Corsi block tapping task [22]. Participants had to remember a sequence of spatial positions previously shown by the experimenter in a wooden board comprising nine positions.

Visuo-spatial active task: Mental pathways. We adapted a procedure used by [25] for a span presentation. Participants had to imagine matrices of varying 


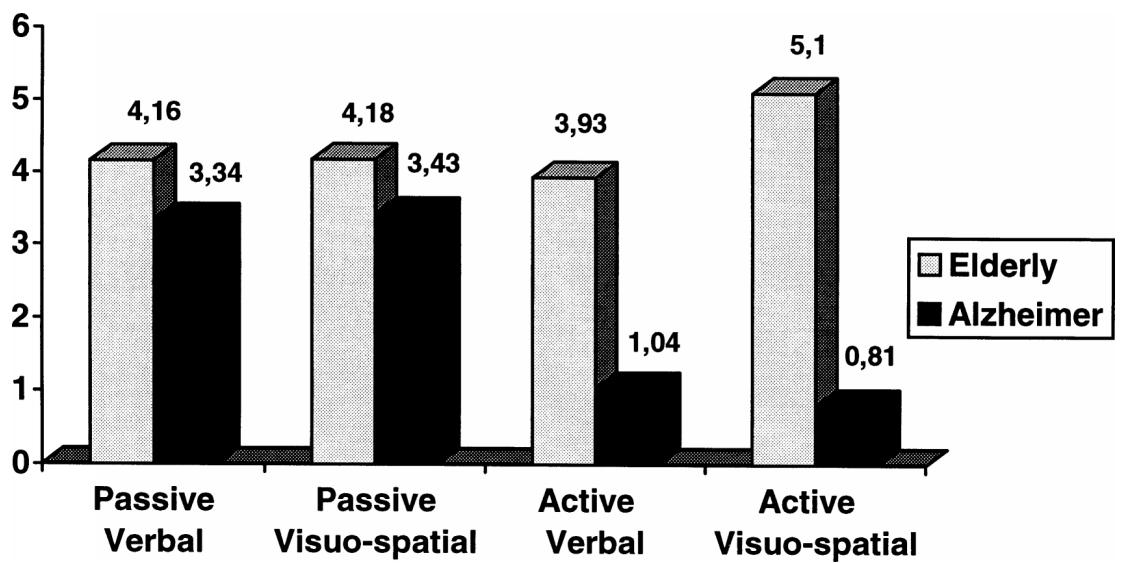

Fig. 1. Mean performance (i.e., span values) of elderly and DAT subjects in the four tasks.

complexity and then generate a pathway following a series of directions (i.e., left, right, forward, backward). The starting point was always the square at the bottom left corner. Directions were read by the experimenter at a 2-sec. rate. After the instructions, participants were presented with a blank matrix and they had to point to the square indicating the final position of the pathway.

\section{Results}

For each task, span values were taken as the mean of the three longest sequences performed correctly. Performance for each task has been analysed separately and four one-way ANOVAs were carried out with group as between-subject variable (elderly and DAT patients). In all tasks, elderly subjects performed better than DAT patients (see Fig. 1), leading to significant differences apart from the case of the visuospatial passive task in which the value just approached significant level: $F(1,30)=8.44, M s e=0.62$, $p<0.01$ for the verbal passive task; $F(1,30)=4.06$, $M s e=1.10, p=0.05$ for the visuo-spatial passive task; $F(1,30)=32.72$, Mse $=5.17, p<0.001$ for the verbal active task; $F(1,30)=16.92$, Mse $=$ $8.69, p<0.001$ for the visuo-spatial active task.

Though the DAT group performance was lower than controls in all tasks, it is also evident that their performance was selectively poor in the active tasks (see Fig. 1). Differences between elderly and DAT participants were larger in tasks requiring to manipulate and actively process experimental stimuli: This is evident in the analyses of effect sizes that allow a direct comparison between passive and active tasks in the two groups of participants (see Table 1). The effect
Table 1

Measures of effect size of the main effect of group in the four tasks. Values are calculated following Cohen's method [5] as reported in [17].

\begin{tabular}{lc}
\hline & Effect size \\
\hline Passive tasks & \\
Verbal & 1.04 \\
Visuo-spatial & 0.71 \\
\hline Active tasks & \\
Verbal & 1.82 \\
Visuo-spatial & 1.46 \\
\hline
\end{tabular}

sizes of the group effects in the active tasks were approximately double the correspondent level for passive tasks, in both verbal and visuo-spatial tasks.

\section{Discussion}

This study showed that DAT patients at an early stage of the disease performed significantly lower than a control group of healthy elderly in the complete set of working memory tasks that we used. However, their deficit was maximised in tasks requiring active manipulation of the material. Increasing levels of active processing determined a dramatic drop in performance in the experimental group, regardless of the type of material presented: verbal and visuo-spatial tasks produced a similar pattern of performance.

This active processing effect cannot be considered as a simple measure of complexity, in that more complex tasks produce more visible deficits. In fact, by using a span procedure, this variable was equally manipulated in all tasks: The span level represents the highest level of complexity that participants were able to deal with. The mean score of each subject is the 
longest span sequence that he/she was able to correctly reproduce or elaborate. Thus, within each subject, the trials that were used to measure performance in the four experimental tasks do represent an equal level of complexity, associated with equal levels of experienced difficulty. The capacity limitations of the system were reached in all tasks but DAT patients showed a dramatic limitation in the active tasks. This deficit indicates a qualitative difference between independent processes, thus excluding the possibility of interpreting this distinction in terms of a quantitative difference between more or less complex task.

Although the levels of complexity/difficulty are clearly matched in the four tasks that we used, this is obviously a critical point in addressing the distinction between passive storage and active processing. To explore this issue in more detail, Vecchi and Richardson [27] developed an experimental task in which the same material and scoring procedure are used for both passive and active tasks, thus allowing a direct comparison between the two tasks. Participants are presented with fragmented pictures of objects in a jumbled configuration: they are required both to recompose the original picture by imaging to place each fragment in the correct positions of a response grid, and then to recall the original jumbled configuration. The temporal sequencing of this task determined a passive task that was significantly more complex than the active one. Since preliminary tests excluded the possibility to use this procedure with DAT patients, the research by [27] was carried out with young and elderly participants only. Results confirmed that passive storage and active processes are (a) independent abilities, and (b) not connected with complexity levels: In fact, elderly people did find the active tasks easier but, at the same time, they were also selectively impaired in these tasks when compared to the younger groups.

A further confirmation of the hypothesis of a dissociation between passive storage and active processing components of working memory comes from neuropsychological and neuroimaging findings. Cornoldi and colleagues [6] have described two cases of children with nonverbal learning difficulties showing a double dissociation between the ability to store visuo-spatial information and the ability to manipulate, transform or integrate visuo-spatial material. While one of child performed flawlessly in the passive tasks and showed an impairment in the execution of the active tasks, the other child presented the opposite pattern. Thus, a preserved ability to manipulate and integrate information could be associated with a specific impairment in the short-term storage of information. In line with these data, a review of recent findings showed that different frontal regions are activated for short-term storage and for manipulation and transformation of information [20].

Overall, these data suggest that active tasks are highly sensitive to the detection of working memory deficits even at an early stage of DAT, in accordance with previous research indicating that the amount of active processing required by a task is a critical variable in predicting performances in working memory tasks (see [24]). The deterioration in working memory functions and, more specifically, a reduction in central processing functions is a diagnostic sign of DAT (e.g., $[2,4])$.

These findings favour the hypothesis that central processing functions may be identified by the specific characteristics of the task (i.e., the amount of active processing) rather than interpreted in terms of supervising or controlling mechanisms only. In addition, this evidence points to the importance of distinguishing, within the working memory model, between modality-specific subsystems (i.e., verbal vs. visuospatial) as well as between process-specific subsystems (i.e., passive vs. active).

\section{Acknowledgements}

The authors wish to thank Dr. Osvaldo Scarpino for his permission to test Alzheimer patients in his care. We thank Cesare Cornoldi, Luisa Girelli, Claudio Luzzatti and Sara Mondini for their help in the design of this research and their comments on an earlier version of this work. We also thank John Richardson for his statistical advice and two anonymous reviewers for their constructive comments.

\section{References}

[1] American Psychiatric Association, Diagnostic and statistic manual of mental disorders, DSM IV. American Psychiatric Association, Washington, 1994.

[2] A.D. Baddeley, S. Bressi, S. Della Sala, R.H. Logie and H. Spinnler, The decline of working memory in Alzheimer's disease, Brain 114 (1991), 2521-2542.

[3] A.D. Baddeley and G.J. Hitch, Working memory, in: The psychology of learning and motivation, Vol. VIII, G. Bower, ed., Academic Press, New York, 1974, pp. 47-90.

[4] A.D. Baddeley, R.H. Logie, S. Bressi, S. Della Sala and H. Spinnler, Dementia and working memory, Quarterly Journal of Experimental Psychology 38A (1986), 603-618.

[5] J. Cohen, Statistical power analysis for the behavioral sciences, Academic Press, New York, 1969. 
[6] C. Cornoldi, F. Rigoni, A. Venneri and T. Vecchi, Passive and Active processes in visuo-spatial memory: Double dissociation in developmental learning disabilities, Brain and Cognition 43 (2000).

[7] A. Cornoldi and T. Vecchi, Mental imagery in blind people: The role of passive and active visuo-spatial processes, in: Touch, representation, and blindness, M. Heller, ed., Oxford University Press, Oxford, 2000, pp. 143-181.

[8] M.J. Farah, K.M. Hammond, D.N. Levine and R. Calvanio, Visual and spatial mental imagery: Dissociable systems of representation, Cognitive Psychology 20 (1988), 439-462.

[9] S.E. Folstein, M.F. Folstein and P.R. McHugh, 'Mini-mental State': A practical method for grading the cognitive state of patients for the clinician, Journal of Psychiatric Research 12 (1975), 189-198.

[10] E. Grober and M.J. Sliwinsky, Dual-task performance in demented and non-demented elderly, Journal of Clinical and Experimental Neuropsychology 13 (1991), 667-676.

[11] J.R. Hanley, A.W. Young and N.A. Pearson, Impairment of the visuo-spatial sketch pad, Quarterly Journal of Experimental Psychology 43A (1991), 101-125.

[12] M.D. Kopelman, Rates of forgetting in Alzheimer-type dementia and Korsakoff's syndrome, Neuropsychologia 23 (1985), 623-628.

[13] C. Luzzatti, T. Vecchi, D. Agazzi, M. Cesa-Bianchi and C. Vergani, A neurological dissociation between preserved visual and impaired spatial processing in mental imagery, Cortex 34 (1998), 461-469.

[14] R.G. Morris, Short-term forgetting in senile dementia of the Alzheimer's type, Cognitive Neuropsychology 3 (1986), 77 97.

[15] R.G. Morris, Attentional and executive dysfunction, in: The cognitive neuropsychology of Alzheimer-type dementia, R.G. Morris, ed., Oxford University Press, Oxford , 1996, pp. 4970.
[16] R.G. Morris and M.D. Kopelman, The memory deficits in Alzheimer-type dementia: A review, Quarterly Journal of Experimental Psychology 38A (1986), 575-602.

[17] J.T.E. Richardson, Measures of effect size, Behavior Research Methods, Instruments, \& Computers 28 (1996), 1222.

[18] T.A. Salthouse, Theoretical perspectives on cognitive aging, Lawrence Erlbaum Associates, Hillsdale, 1991.

[19] T. Shallice, From neuropsychlogy to mental structure, Cambridge University Press, Cambridge, 1988.

[20] E.E. Smith and J. Jonides, Storage and executive processes in the frontal lobes, Science 283 (1999), 1657-1661.

[21] H. Spinnler, S. Della Sala, R. Bandera and A.D. Baddeley, Dementia, ageing, and the structure of human memory, Cognitive Neuropsychology 5 (1988), 193-211.

[22] H. Spinnler and G. Tognoni, Standardizzazione e taratura italiana di test neuropsicologici. Italian Journal of Neurological Sciences supplemendum 8 (1987).

[23] G. Vallar and A.D. Baddeley, Fractionation of working memory: Neuropsychological evidence for a phonological shortterm store, Journal of Verbal Learning and Verbal Behavior 23 (1984), 151-161.

[24] T. Vecchi and C. Cornoldi, Passive storage and active manipulation in visuo-spatial working memory: Further evidence from the study of age differences, European Journal of Cognitive Psychology 11 (1999), 391-406.

[25] T. Vecchi M. Monticelli and C. Cornoldi, Visuo-spatial working memory: structures and variables affecting a capacity measures, Neuropsychologia 33 (1995), 1549-1564.

[26] T. Vecchi and J.T.E. Richardson, Active processing in visuospatial working memory. Cahiers de Psychologie Cognitive 19 (2000), 3-32.

[27] T. Vecchi and J.T.E. Richardson, Processing and complexity effects in working memory (submitted). 


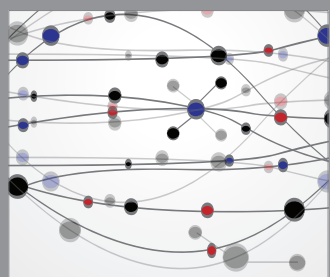

The Scientific World Journal
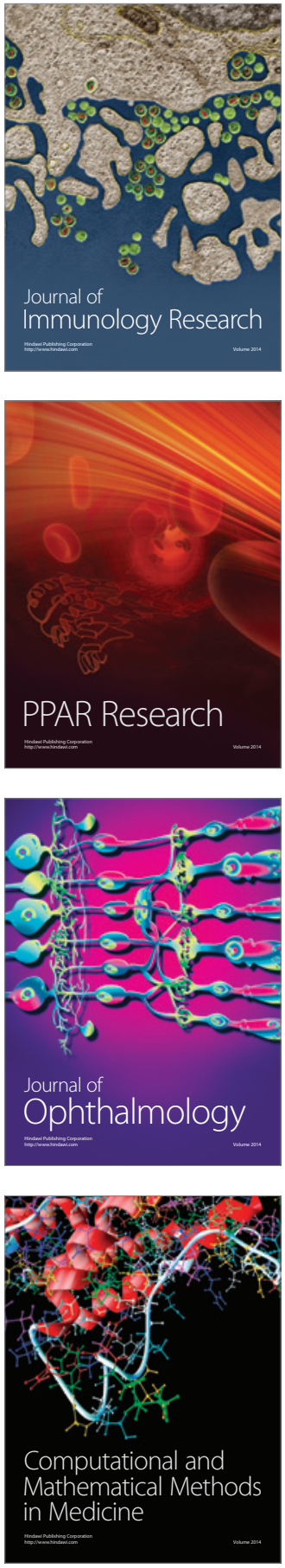

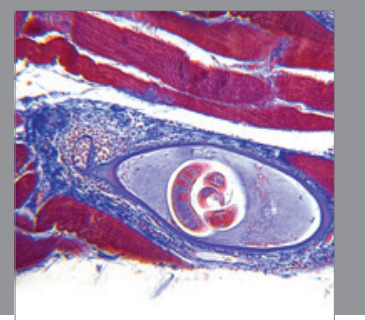

Gastroenterology

Research and Practice
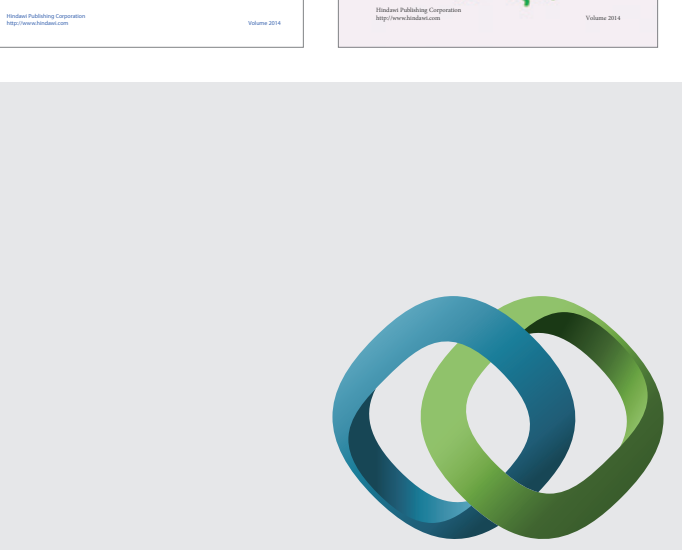

\section{Hindawi}

Submit your manuscripts at

http://www.hindawi.com
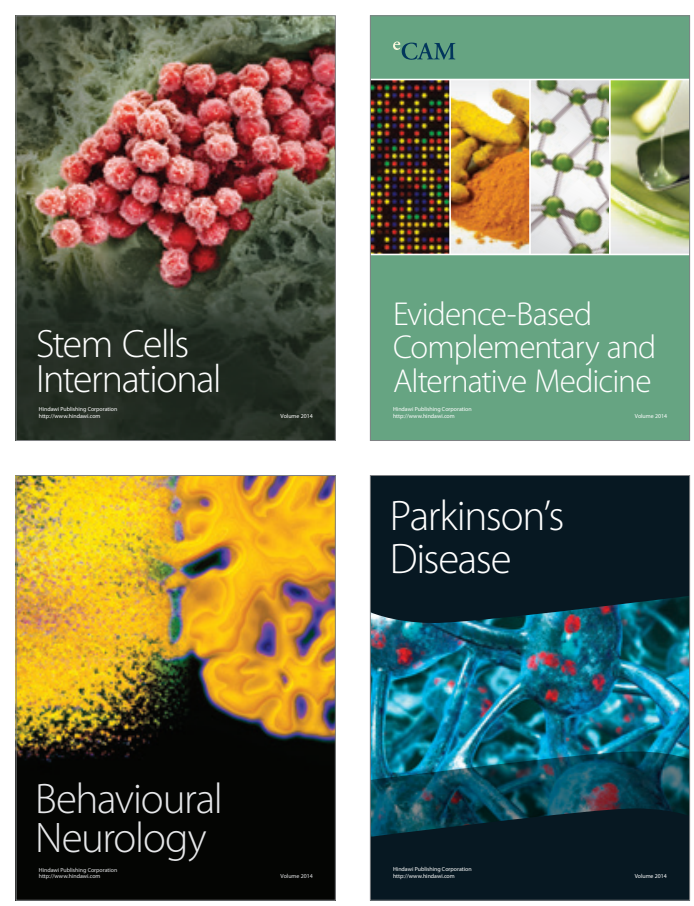

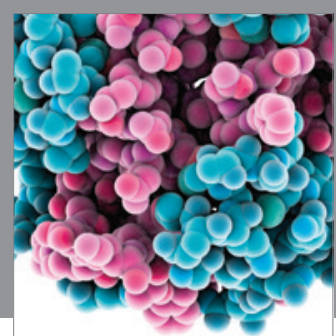

Journal of
Diabetes Research

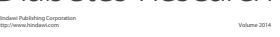

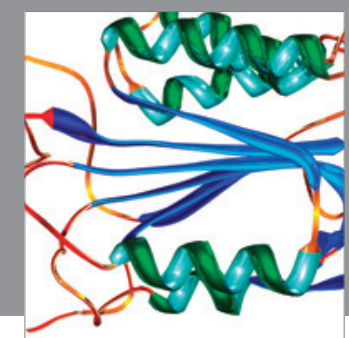

Disease Markers
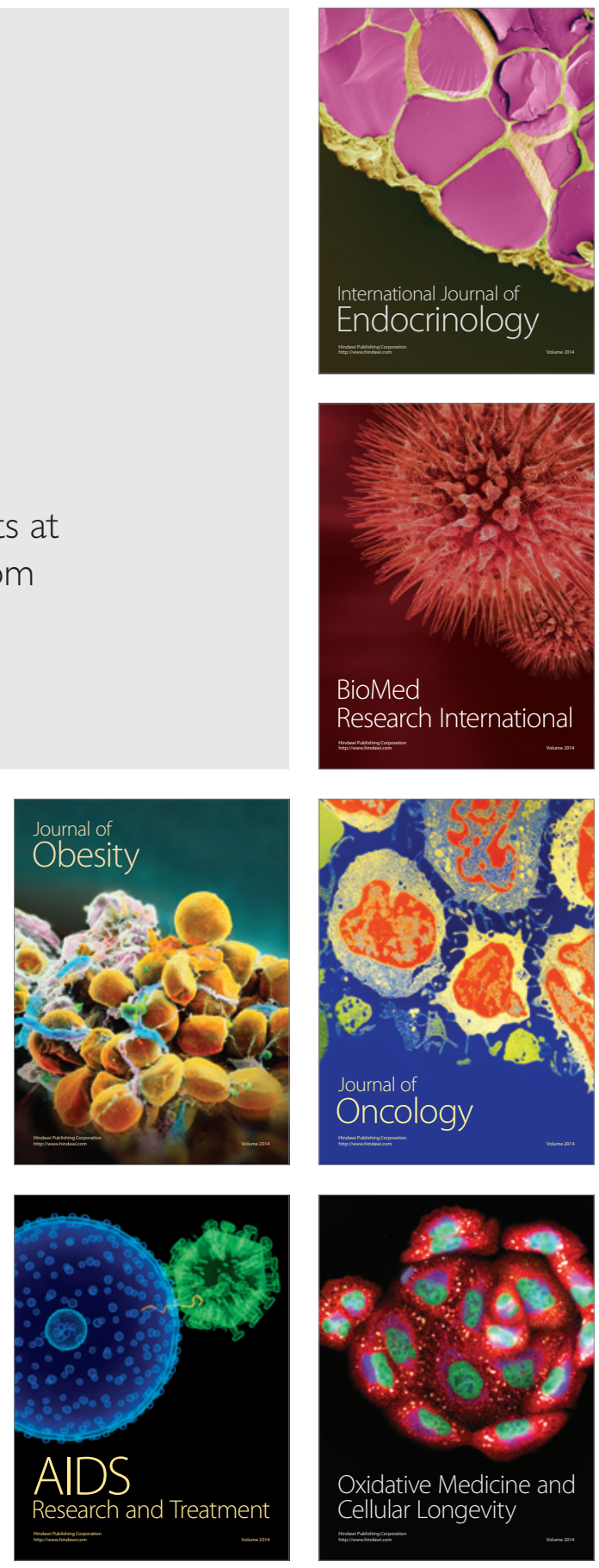